\title{
2D materials for conducting holes from grain boundaries in perovskite solar cells
}

\author{
Peng You $\mathbb{1}^{1,2}$, Guanqi Tang ${ }^{1}$, Jiupeng Cao ${ }^{1}$, Dong Shen ${ }^{3}$, Tsz-Wai $\mathrm{Ng}^{3}$, Zafer Hawash ${ }^{4}$, Naixiang Wang $\mathbb{D}^{1}$,

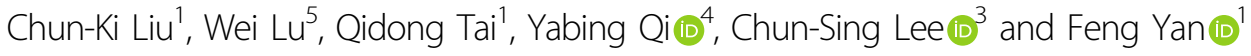

\begin{abstract}
Grain boundaries in organic-inorganic halide perovskite solar cells (PSCs) have been found to be detrimental to the photovoltaic performance of devices. Here, we develop a unique approach to overcome this problem by modifying the edges of perovskite grain boundaries with flakes of high-mobility two-dimensional (2D) materials via a convenient solution process. A synergistic effect between the 2D flakes and perovskite grain boundaries is observed for the first time, which can significantly enhance the performance of PSCs. We find that the 2D flakes can conduct holes from the grain boundaries to the hole transport layers in PSCS, thereby making hole channels in the grain boundaries of the devices. Hence, 2D flakes with high carrier mobilities and short distances to grain boundaries can induce a more pronounced performance enhancement of the devices. This work presents a cost-effective strategy for improving the performance of PSCs by using high-mobility 2D materials.
\end{abstract}

\section{Introduction}

Perovskite solar cells (PSCs) based on organic-inorganic halide perovskites have been increasingly studied in recent years. Since the first report in 2009 of a perovskite material used in solar cells ${ }^{1}$, the power conversion efficiencies (PCEs) of PSCs have now reached a certified value higher than those of solar cells based on multi-crystalline $\mathrm{Si}$, cadmium telluride or copper indium gallium diselenide, according to the efficiency chart provided by the National Renewable Energy Laboratory $(\mathrm{NREL})^{2}$. Organic-inorganic halide perovskites have shown many advantages over conventional semiconductors for photovoltaics, including long carrier lifetimes, high light absorption, easy processing and low fabrication $\operatorname{cost}^{3-11}$. Therefore, PSCs are promising for use in practical applications in the future.

\footnotetext{
Correspondence: Feng Yan (apafyan@polyu.edu.hk)

'Department of Applied Physics, The Hong Kong Polytechnic University, Hung Hom, Kowloon, Hong Kong, China

${ }^{2}$ College of New Materials and New Energies, Shenzhen Technology University, 518118 Shenzhen, China
}

Full list of author information is available at the end of the article
Grain boundaries (GBs) in PSCs have been found to be detrimental to the photovoltaic performance of devices ${ }^{12}$. Numerous papers have reported that defects in perovskite GBs should be passivated by suitable materials, such as quaternary ammonium halide ${ }^{13}$, fullerene derivatives ${ }^{14-16}$ and $\mathrm{CH}_{3} \mathrm{NH}_{3} \mathrm{I}$ (MAI) ${ }^{17}$, to alleviate carrier recombination and consequently improve device performance. Here, we report a novel method to overcome the drawback of perovskite GBs without passivating defects. Several 2D materials, including black phosphorus (BP), $\mathrm{MoS}_{2}$ and graphene oxide (GO), are specifically modified on the edge of perovskite GBs by a solution process. These 2D materials have high carrier mobilities, ultrathin thicknesses and smooth surfaces without dangling bonds ${ }^{18-22}$. The PCEs of the devices are substantially enhanced by the 2D flakes, in which BP flakes can induce the highest relative enhancement of $\sim 15 \%$. Notably, we find that under certain conditions, GBs modified with 2D materials are favourable for device performance. Therefore, a synergistic effect between 2D flakes and perovskite GBs is observed for the first time. Although nanotechnology using 2D materials in PSCs has been reported in some papers ${ }^{20,23-26}$, the synergistic effect between $2 \mathrm{D}$

\section{(c) The Author(s) 2021}

(c) (i) Open Access This article is licensed under a Creative Commons Attribution 4.0 International License, which permits use, sharing, adaptation, distribution and reproduction cc) in any medium or format, as long as you give appropriate credit to the original author(s) and the source, provide a link to the Creative Commons license, and indicate if changes were made. The images or other third party material in this article are included in the article's Creative Commons license, unless indicated otherwise in a credit line to the material. If material is not included in the article's Creative Commons license and your intended use is not permitted by statutory regulation or exceeds the permitted use, you will need to obtain permission directly from the copyright holder. To view a copy of this license, visit http://creativecommons.org/licenses/by/4.0/. 
flakes and perovskite GBs has not been reported until now. To better understand the underlying mechanism of the above effect, device simulation is conducted by using commercial software ${ }^{27}$. The hole conduction processes from GBs to 2D flakes in PSCs are clearly demonstrated, showing that the GBs and 2D flakes all act as hole channels in the devices. The simulation results confirm that the performance enhancement induced by BP is higher than that induced by the other 2D materials because $\mathrm{BP}$ has the highest hole mobility ${ }^{28-34}$. In addition, the modification of $2 \mathrm{D}$ flakes on the perovskite grains away from GBs has little effect on device performance, indicating that the synergistic effect of 2D flakes and perovskite GBs is essential to the performance enhancement in our devices.

\section{Results}

\section{Modification of BP flakes on perovskite films}

First, we modified the surfaces of $\mathrm{CH}_{3} \mathrm{NH}_{3} \mathrm{PbI}_{3}$ $\left(\mathrm{MAPbI}_{3}\right)$ perovskite active layers in PSCs with BP flakes by a solution process. BP has recently emerged as a promising 2D semiconducting material for various applications owing to its tunable direct bandgap and high carrier mobility ${ }^{28}$. A BP dispersion solution was prepared using an ultrasonication method from BP crystals (Fig. S1a) ${ }^{35}$. Anhydrous isopropanol (IPA) was chosen as an orthogonal solvent for the BP dispersion to be coated on perovskite films. BP flakes were first coated on flat Si substrates and characterized with atomic force microscopy (AFM) (Fig. S1b). The average size and thickness of the BP flakes are estimated to be $39 \pm 19 \mathrm{~nm}$ and $4.3 \pm$ $2.0 \mathrm{~nm}$, respectively (Fig. S1d, e). The high-resolution transmission electron microscopy (HRTEM) image of a BP flake confirms the orthorhombic crystal structure of BP (Fig. S2). Ultraviolet photoelectron emission spectra (UPS) of the BP thin films on silicon substrates were obtained (Fig. S3a). The valence band maximum (VBM) of $\mathrm{BP}$ is found to be $\sim-5.32 \mathrm{eV}$. Based on the PL spectrum of BP films (Fig. S4), the bandgap of BP flakes is estimated to be approximately $1.53 \mathrm{eV}$. Therefore, the conduction band minimum $(\mathrm{CBM})$ of the $\mathrm{BP}$ flakes is calculated to be $-3.79 \mathrm{eV}$.

PSCs were fabricated with a device configuration of glass/ fluorine-doped tin oxide (FTO)/compact $\mathrm{TiO}_{2}\left(\mathrm{c}-\mathrm{TiO}_{2}\right) /$ mesoporous $\mathrm{TiO}_{2}\left(\mathrm{mp}-\mathrm{TiO}_{2}\right) / \mathrm{MAPbI}_{3} / \mathrm{BP} / 2,2^{\prime}, 7,7^{\prime}$-tetrakis-(N,N-di-p-methoxyphenylamine)-9,9'-spirobifluorene (spiro-OMeTAD)/Au, as shown in Fig. 1a. $\mathrm{MAPbI}_{3}$ perovskite films were prepared by an anti-solvent-assisted crystallization method ${ }^{36}$. BP flakes were modified on perovskite layers by spin coating the BP dispersion solution on their surfaces. Figure $1 \mathrm{~b}$ shows the band structure of the PSCs, where some of the energy levels are derived from the UPS spectra (Fig. S3) and others are taken from the literature $^{37,38}$. It is notable that the VBM of BP $(-5.32 \mathrm{eV})$ matches very well with those of $\mathrm{MAPbI}_{3}$ perovskite $(-5.50 \mathrm{eV})$ and spiro-OMeTAD $(-5.15 \mathrm{eV})$. The cascade band structure of the multilayer device is favourable for the separation of electrons and holes and can reduce the carrier recombination rate at the interface between perovskite and hole transport materials (HTMs).

Figure 1c shows the representative current density-voltage $(J-V)$ curves of PSCs without (control device) and with BP flakes modified on the perovskite surfaces. The amount of BP flakes on the surfaces was controlled by coating the solution for different times. The control devices exhibit only a moderate PCE (17.97\%), while a clearly improved efficiency $(19.85 \%)$ is obtained when the BP solution is coated one time. The PCE further improves to $20.32 \%$ when BP is spin-coated two times. However, the device performance cannot be further improved when BP is coated three times. The corresponding photovoltaic parameters for champion devices and the average values for 30 devices are listed in Table S1.

Figure 1d shows the external quantum efficiency (EQE) spectra of the above PSCs. In comparison with the control devices, a remarkable increase in EQE can be observed on the BP-modified devices over the whole wavelength range (from 300 to $800 \mathrm{~nm}$ ), which explains the enhanced $J_{\mathrm{sc}}$ in the $J-V$ characteristics. The integrated current density based on the highest EQE spectrum (two coats of BP) is $22.5 \mathrm{~mA} \mathrm{~cm}^{-2}$ (Fig. S5a), which is very close to the shortcircuit current density presented in the $J-V$ curve of the device.

Figure 1e shows the histogram statistics of PCEs for 30 devices fabricated with BP modification (two coats) and 30 control devices. The average PCE of the PSCs is improved by the BP flakes from 16.94 to $19.54 \%$ with a relative enhancement of $15.3 \%$. By applying a voltage bias at the maximum power point $(0.935 \mathrm{~V})$ to the champion device, a stabilized photocurrent of $21.30 \mathrm{~mA} \mathrm{~cm}^{-2}$ and an efficiency of $\sim 19.92 \%$ are obtained (Fig. S5b), which is equal to the average value of the PCEs obtained from the reverse $(20.32 \%)$ and forward (19.53\%) scans of the $J-V$ curves. We also notice that the hysteresis of the $J-V$ curves is greatly reduced due to the incorporation of $\mathrm{BP}$ flakes (Fig. S5c and Table S2). Control experiments indicate that the solvent IPA has no obvious effect on the performance of PSCs (Fig. S5d).

\section{Effect of BP flakes on perovskite grain boundaries}

To shed light on the effect of BP flakes, the morphology of $\mathrm{MAPbI}_{3}$ perovskite films was characterized with scanning electron microscopy (SEM), as shown in Fig. 2. The SEM images demonstrate that the perovskite films have an average grain size of $\sim 300 \mathrm{~nm}$ without pinholes. It is notable that the coverages of BP flakes on the film surfaces are only $4 \%, 8$ and $10 \%$ for 1,2 and 3 coats of the BP solution, respectively. Although the coverages are rather 
a
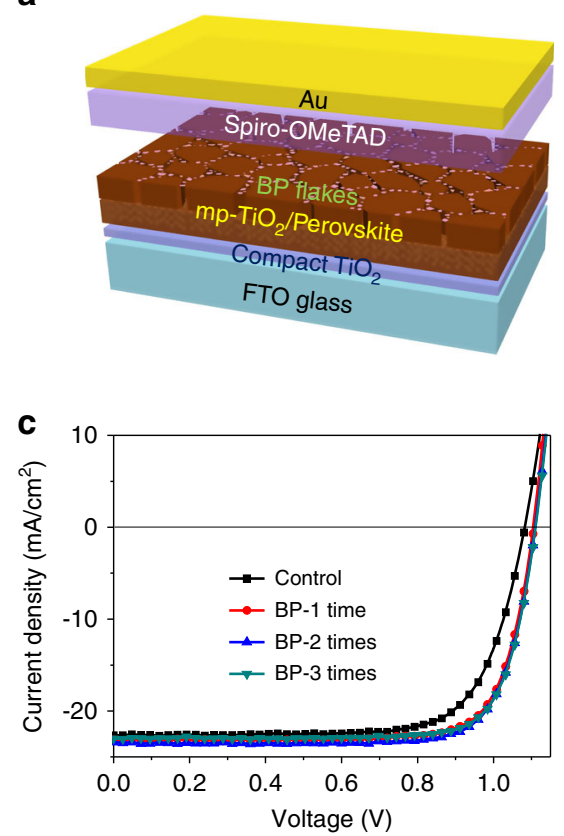

e

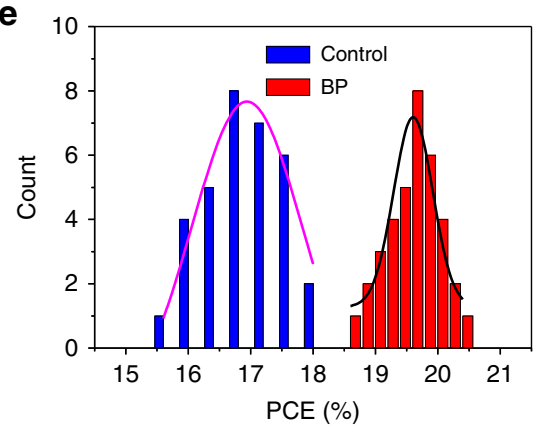

b

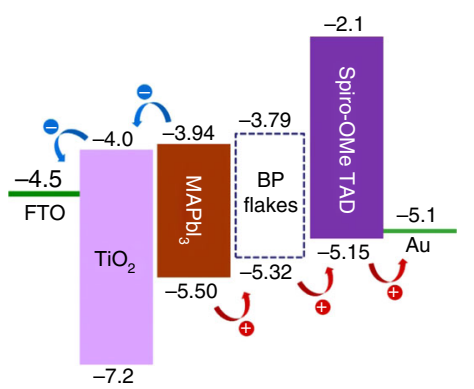

d
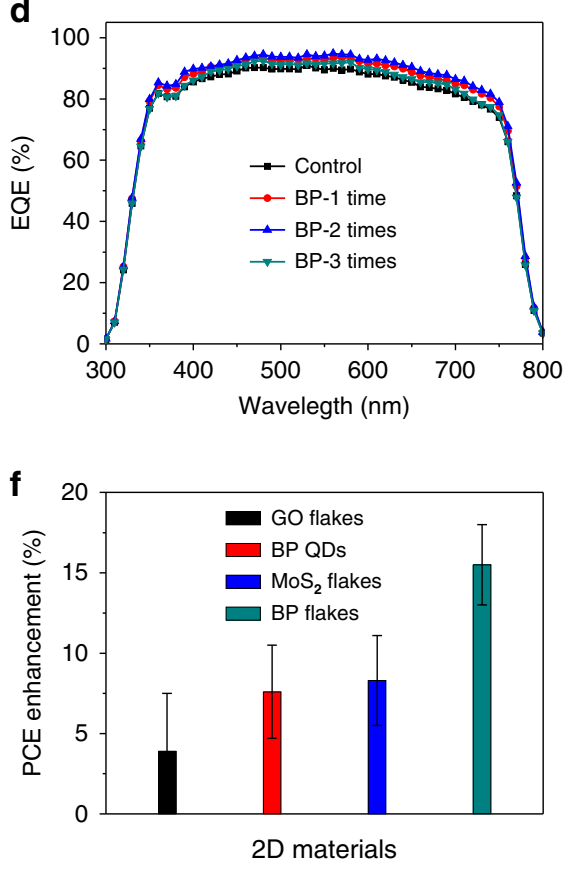

Fig. 1 Device structure and photovoltaic performance of $\mathrm{MAPbl}_{3}$ PSCs. $\mathbf{a}, \mathbf{b}$ Structural schematic diagram and energy-level band diagram of a BP-modified PSC with a normal structure. $\mathbf{c}, \mathbf{d}$ Current density-voltage $(J-V$ curves (reverse scan) and external quantum efficiency (EQE) spectra of PSCs without (control) and with BP (1-3 times) deposited on the perovskite film surface. e Histogram of the power conversion efficiencies (PCEs) of PSCs without (control) and with the BP modification (2 coats of BP). The PCEs are derived from the reverse scans of the J-V curves. $\mathbf{f}$ Average PCE enhancement after the modification of perovskite films with different $2 \mathrm{D}$ materials. The error bar corresponds to the standard error for 30 devices

low, almost all the BP flakes are located on the GBs of the perovskite films. Notably, the space charge limited current (SCLC) characterizations of hole-only and electron-only devices (Fig. S6) confirm that the introduction of BP flakes at perovskite GBs has little effect on the electron and hole mobilities of the perovskite layer. Therefore, we believe the effect of BP flakes on PSCs is mainly due to the modification of GBs. The existence of BP flakes on the perovskite surface is also confirmed by energy-dispersive X-ray spectroscopy (EDX) mapping of phosphorene (Fig. S7). Notably, BP flakes on the GBs cannot passivate defects because they are physically absorbed on the edge of GBs, and no chemical bond can be formed with the perovskite layer, which is confirmed by Fourier transform infrared (FTIR) spectroscopy measurements (see Fig. S8).
To determine why the BP flakes are coated on the GBs only, we measured the contact angle of IPA on perovskite films with different crystallinities (Fig. S9). The average contact angles are $8^{\circ}, 16^{\circ}$ and $27^{\circ}$ for the amorphous, polycrystalline and single-crystal perovskite surfaces, respectively, indicating that the contact angle of IPA decreases with the decreasing crystallinity of perovskite films. The surface energy of IPA on a perovskite film is related to the contact angle $\theta$ provided by Young's equation $^{39}$ :

$$
\cos \theta=\frac{\gamma_{S V}-\gamma_{S L}}{\gamma_{L V}}
$$

where $\gamma_{S V}, \gamma_{S L}$ and $\gamma_{L V}$ are the interfacial tensions of the solid-vapor, solid-liquid, and liquid-vapor interfaces, 


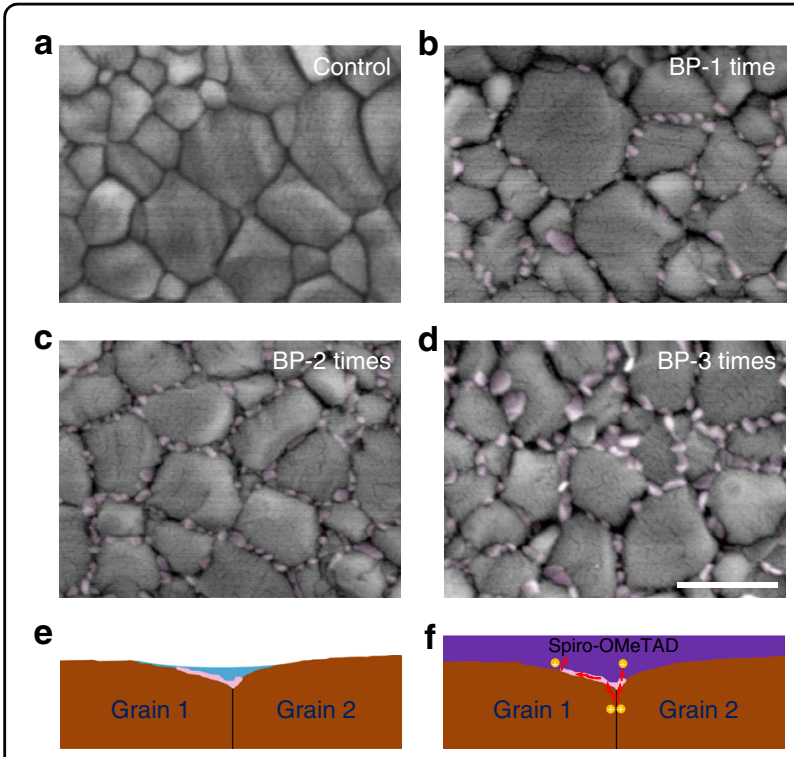

Fig. 2 Morphology of the BP-modified $\mathrm{MAPbl}_{3}$ perovskite films. a-d SEM images of the perovskite films without and with 1-3 coats of BP flakes. The scale bar is $500 \mathrm{~nm}$. e Schematic diagram of the grain boundary (GB) area when the BP dispersion in IPA was spin coated on the perovskite films. $\mathbf{f}$ Schematic diagram of the GB area after the deposition of BP and spiro-OMeTAD. Holes can transfer from the GB to the $\mathrm{BP}$ flake and then transport along the flake to the hole transport material spiro-OMeTAD

respectively, and $\gamma_{S V}-\gamma_{S L}$ is the adhesion tension. Therefore, the adhesion tension increases with decreasing crystallinity. On the other hand, GB grooves can be formed during the crystallization process due to the diffusion of ions away from the boundaries ${ }^{40}$. Therefore, during the spin-coating process, the $\mathrm{BP}$ solution tends to move to GB regions, as shown in Fig. 2e, resulting in BP flakes mainly depositing on GBs after the evaporation of the IPA solvent.

Based on the above results, we present a schematic diagram in Fig. 2f to illustrate the mechanism for the performance enhancement induced by BP flakes in a PSC. Under light illumination, photo-generated holes tend to move to the GB and generate hole currents across BP flakes. Because BP has a much higher hole mobility $\left(\sim 1000 \mathrm{~cm}^{2} \mathrm{~V}^{-1} \mathrm{~s}^{-1}\right)$ than spiro-OMeTAD $\left(\sim 10^{-4} \mathrm{~cm}^{2} \mathrm{~V}^{-1} \mathrm{~s}^{-1}\right)$, BP flakes can conduct hole currents from GBs more efficiently. This model is also based on the assumption that the hole current density along a GB is higher than that in the adjacent grains due to band bending when near the GB (Fig. S10). To confirm this effect, we characterized our perovskite films on $\mathrm{TiO}_{2}$ by using c-AFM and find that the photo-induced hole current density at a GB is normally higher than that on adjacent grain surfaces, which is consistent with the results in the literature ${ }^{41}$. Moreover, the band banding that occurs close to a GB can enhance electron-hole separation due to the built-in electric field in this region. Therefore, GBs act as hole channels in perovskite active layers, and BP flakes can enhance hole transfer from GBs in the devices to improve device performance.

In addition, we are keen to know whether BP flakes can conduct electrons in PSCs to improve device performance. Hence, PSCs with an inverted structure of glass/ITO/poly [bis(4-phenyl)(2,4,6-trimethylphenyl)amine] (PTAA)/MA $\mathrm{PbI}_{3} / \mathrm{BP} /$ phenyl-C61-butyric acid methyl ester (PCBM)/ bathocuproine (BCP)/Ag were prepared ${ }^{42}$. BP flakes were modified on the perovskite surfaces under the same conditions as the above experiments before coating the PCBM electron transport layers (ETLs). However, the performance of the inverted PSCs is not enhanced by the addition of BP flakes (Fig. S11), which can be attributed to the following two reasons. First, GBs in perovskite films can only conduct holes; thus, the modification of BP flakes on GBs beneath the ETL cannot facilitate the transfer of electrons in these devices. Second, the band structure of BP flakes is not favourable for electron transfer in these devices since the CBM level of $\mathrm{BP}$ is $\sim 0.15 \mathrm{eV}$ higher than that of the perovskite layer.

To better understand the effect of BP flakes on $\mathrm{MAPbI}_{3}$ perovskite films, we characterized the steady-state and time-resolved photoluminescence (PL) behaviour of the following three samples: perovskite, perovskite/spiroOMeTAD and perovskite/BP/spiro-OMeTAD films. As shown in Fig. 3a, the perovskite film without any modification exhibits the highest PL peak. The introduction of a spiro-OMeTAD layer on top of perovskite greatly reduces the PL intensity, which is further quenched by the presence of a BP layer, indicating more efficient hole transfer in the perovskite/BP/spiro-OMeTAD film. The time-resolved PL decay curves presented in Fig. 3b show a similar effect. The decay curves are fitted with a biexponential model: $\mathrm{y}(t)=y_{0}+A_{1} e^{-t / \tau_{1}}+A_{1} e^{-t / \tau_{2}}$ (Fig. S12). The derived PL lifetime constants $\left(\tau_{1}\right.$ and $\left.\tau_{2}\right)$ of the bare perovskite are $3.4 \mathrm{~ns}$ and $25.3 \mathrm{~ns}$, which decrease to $1.4 \mathrm{~ns}$ and $9.2 \mathrm{~ns}$ by spiro-OMeTAD and further shorten to $0.8 \mathrm{~ns}$ and $2.9 \mathrm{~ns}$ after the BP modification. The improved PL quenching yield of the BP-modified devices indicates an enhanced hole transfer rate from perovskite to spiroOMeTAD via the BP flakes on GBs. These results are consistent with the improved efficiency of BP-modified PSCs.

To further investigate the charge recombination behaviour of PSCs, we performed electrochemical impedance spectroscopy (EIS) measurements on the devices under light illumination of $100 \mathrm{~mW} \mathrm{~cm}^{-2}$. Figure $3 \mathrm{c}$ shows the EIS spectra of the devices at a bias voltage of $0.80 \mathrm{~V}$. Two distinct regions can be observed in the Nyquist plots, including a high-frequency region correlated with the charge recombination process (large half circle) and a low-frequency region corresponding to the slow dielectric 
a
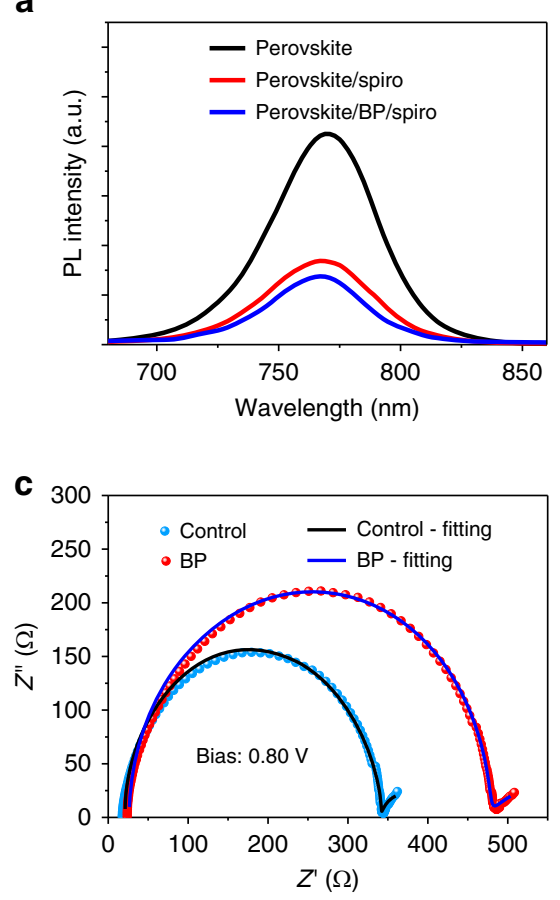

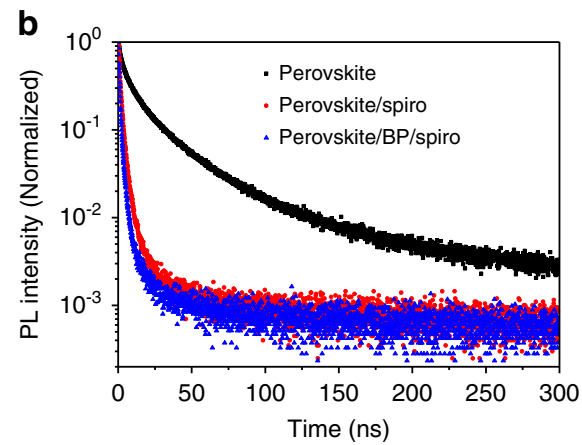

d

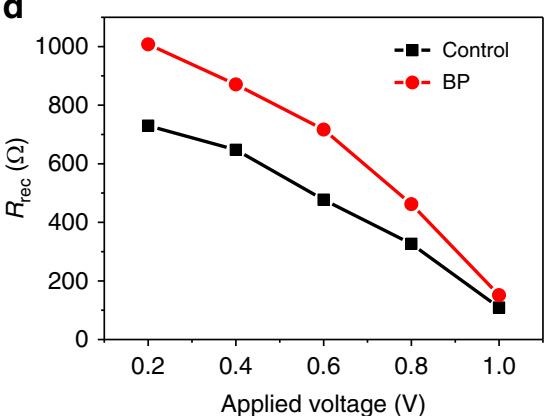

Fig. 3 Optoelectronic characteristics of the $\mathrm{MAPbl}_{3}$ perovskite films and devices. $\mathbf{a}, \mathbf{b}$ Steady state (a) and time-resolved (c) PL spectra of three films, including the perovskite, perovskite/spiro and perovskite/BP/spiro films; the films were deposited on quartz substrates. $\mathbf{c}$ Impedance spectra of the PSCs without (control) and with BP modification (2 coats) measured at a bias voltage of $0.80 \mathrm{~V}$ under light illumination of $100 \mathrm{~mW} \mathrm{~cm}^{-2}$. d Recombination resistance of PSCs derived from impedance spectra at different bias voltages under light illumination of $100 \mathrm{~mW} \mathrm{~cm}-2$

and ionic relaxation in perovskite films (part of the small (ircle $)^{43}$. Figure $3 \mathrm{~d}$ shows the recombination resistances $\left(R_{\text {rec }}\right)$ of the two PSCs at different bias voltages, which are derived from the Nyquist plots (Fig. S13). Both devices show a decreasing $R_{\text {rec }}$ with an increasing bias voltage due to increased carrier densities ${ }^{43,44}$. A high $R_{\text {rec }}$ value and long carrier lifetime can be observed in the BP-modified solar cell at any bias voltage, indicating a slow recombination rate being induced by BP flakes; thus, the improved hole extraction rate from perovskite to spiro-OMeTAD can be attributed to the BP flakes.

The stability of BP-modified PSCs was investigated in air. The statistical data of the PCEs of devices are presented in Fig. S14. The BP-modified PSCs and control devices can maintain $\sim 92$ and $76 \%$ of their initial average PCE after $1000 \mathrm{~h}$, indicating that the devices with BP flakes are more stable than the control devices. This improved stability of the device can be attributed to the BP flakes that partially block the diffusion of outside ions, oxygen and moisture to the $\mathrm{GBs}^{45-47}$. On the other hand, Ahn et al. reported that the GBs in perovskite films are unstable in the simultaneous presence of charge and moisture ${ }^{48}$. BP flakes can efficiently conduct holes and decrease the density of holes accumulated in GBs, which can be another reason for the improved stability of BP-modified devices.

\section{Grain-size effect of perovskite films with the BP modification}

To demonstrate the effect of GBs in PSCs, we prepared $\mathrm{MAPb}_{3}$ perovskite films with different grain sizes by controlling the thermal annealing periods. As shown in Fig. 4, perovskite films with average grain sizes of 200, 250, 300 and $390 \mathrm{~nm}$ were prepared after thermal annealing at $100^{\circ} \mathrm{C}$ for $10,30,60$ and $120 \mathrm{~min}$, respectively. For the devices without the BP flake modification, the device efficiency increases with an increasing grain size (Table S3 and Table S4), which is consistent with the results in the literature $^{42,49}$. After the BP flake modification under the same conditions, the PCEs of the devices dramatically improve. When the average grain size is $\sim 300 \mathrm{~nm}$, the devices show the highest PCE and the maximum relative enhancement. This result indicates that a suitable number of GBs is favourable for the performance of PSCs because the photocurrents in the devices can be conducted out along the GBs (Fig. S10). Notably, devices with smaller grain sizes (200 and $250 \mathrm{~nm}$ ) show lower PCE enhancements when the GBs are modified with BP flakes. We can find the reason from the cross-sectional SEM images of the perovskite films shown in Fig. 4. When the grain size is smaller than $300 \mathrm{~nm}$, the perovskite grains cannot penetrate the films from the bottom to the top. In this case, the GBs in the middle will 


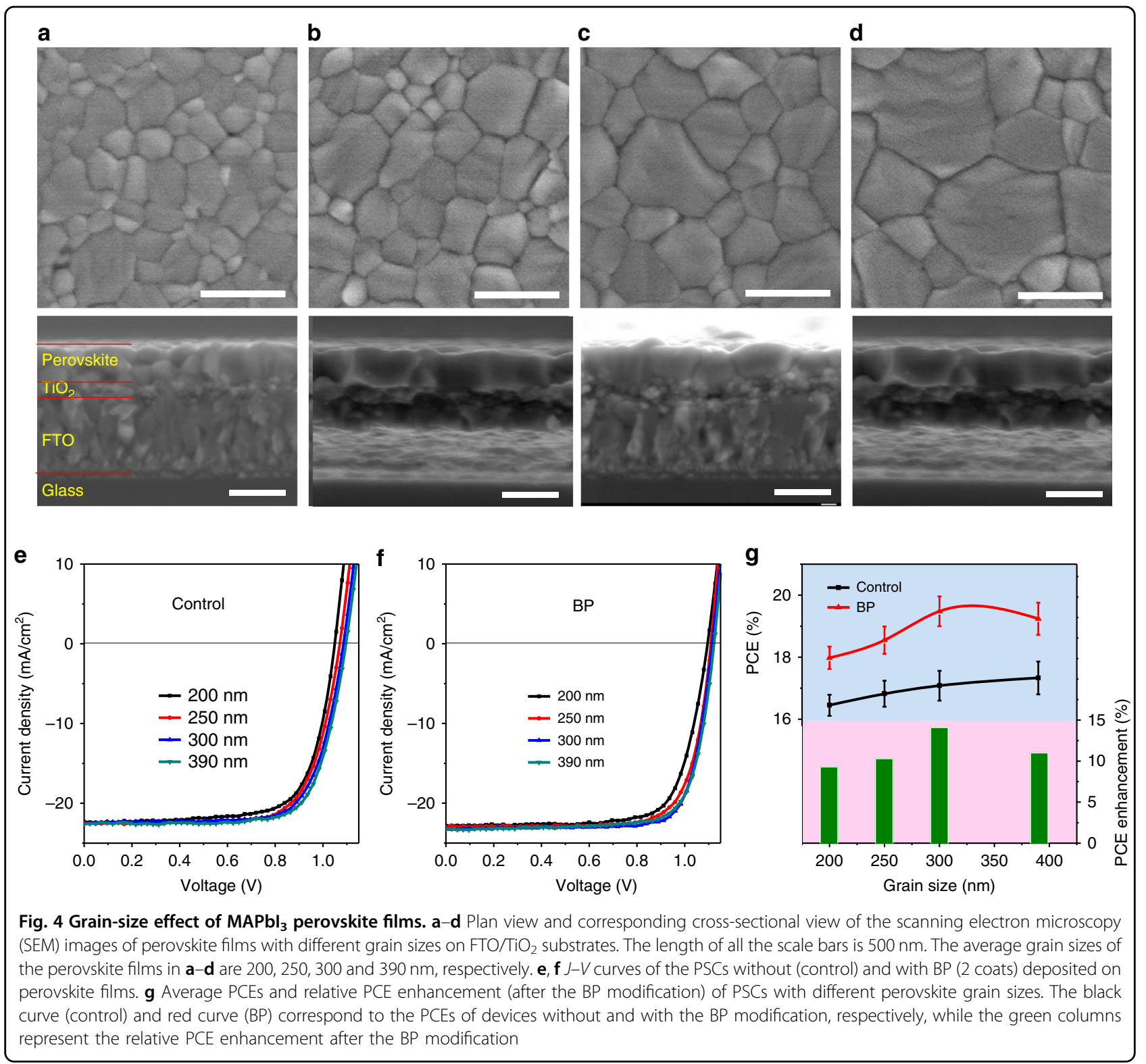

prohibit electron transport in the devices, although they continue to be hole conductors (Fig. S10f) ${ }^{50}$.

\section{Modification of other 2D material flakes}

Solutions of other 2D materials, including $\mathrm{MoS}_{2}, \mathrm{GO}$ and BP quantum dots (BP-QDs), were prepared and modified on the perovskite layers of PSCs with the same procedure ${ }^{51-53}$. As expected, almost all of the $\mathrm{MoS}_{2}$ and GO flakes are located on the perovskite GBs (Fig. S15). The average sizes of the $\mathrm{MoS}_{2}$ and GO flakes are estimated to be $\sim 40 \mathrm{~nm}$. However, BP-QDs are too small to be observed with SEM. The average size of BP-QDs observed with TEM is less than $5 \mathrm{~nm}$ (Fig. S16). It is reasonable to expect that BP-QDs are located on the GBs of perovskite films as well. The $J-V$ curves and photovoltaic parameters of the PSCs modified with $\mathrm{MoS}_{2}$, GO and BP-QDs under optimum coating conditions were characterized (Fig. S17 and Table S5). The photovoltaic performances of the devices are improved by all the 2D materials, and the PCE enhancements are presented in Fig. 1f. Notably, BP flakes can induce better photovoltaic performance than other 2D materials, which can be attributed to BP having the highest carrier mobility. Moreover, BPQDs can only conduct holes at a distance of several nanometres, and thus, their effect is inferior to that of BP flakes.

\section{Device simulation for the effect of 2D flakes}

To better understand the performance enhancement of

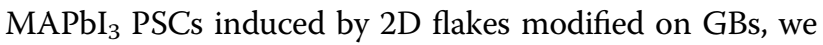



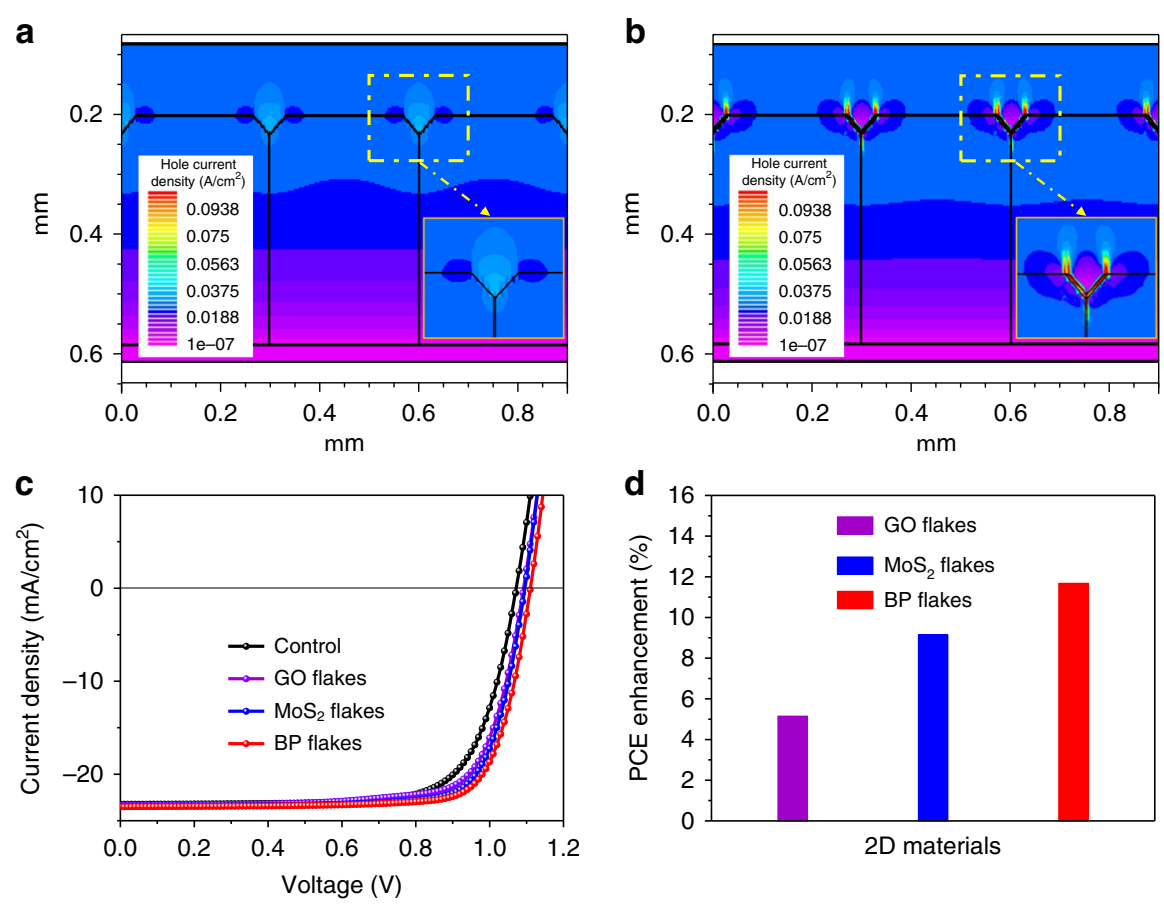

Fig. 5 Device simulation results of the MAPbl $_{3}$ PSCs. $\mathbf{a}, \mathbf{b}$ Hole current density distribution of the simulated PSCs without (a) and with (b) the BP modification. $\mathbf{c}$ Simulated J-V curves of the PSCS modified with different 2D materials. d Relative PCE enhancement of the PSCs after modifications with different $2 \mathrm{D}$ materials

performed a device simulation by using the commercial software Silvaco. A device with a perovskite grain size of $300 \mathrm{~nm}$ was designed for simulation (Fig. S18, Table S6 and Table S7). The GB grooves were assumed to be $30 \mathrm{~nm}$ deep, which is consistent with our AFM measurements. To simulate the effect of BP in the devices, a BP layer was added on the surfaces of these grooves. As shown in Fig. 5, the $J-V$ curves of the devices are simulated before and after coating the BP layer under standard illumination conditions (AM $1.5 \mathrm{G}$, power density: $100 \mathrm{~mW} \mathrm{~cm}^{-2}$ ). It is interesting to find that the simulated performance is very similar to our experimental results (Table S8). After the BP modification, the open-circuit voltage and the fill factor of the device are substantially improved. We can clearly find high hole currents conducted out by the BP flakes from the GBs in Fig. 5b. Here, the hole mobility of BP is $\sim 1000 \mathrm{~cm}^{2} \mathrm{~V}^{-1} \mathrm{~s}^{-1}$, which is 7 orders of magnitude higher than that of spiro-OMeTAD ${ }^{29}$. When we replaced BP with $\mathrm{MoS}_{2}$, which has a hole mobility of $\sim 70 \mathrm{~cm}^{2} \mathrm{~V}^{-1} \mathrm{~s}^{-154-56}$, the PCE enhancement is lower than that provided by BP. We also simulated the effect of GO flakes (hole mobility is assumed to be $\sim 0.015 \mathrm{~cm}^{2} \mathrm{~V}^{-1} \mathrm{~s}^{-1}$ ) on GBs, and a performance enhancement is observed due to the hole conduction of $\mathrm{GO}^{57}$. Our simulation results demonstrate that the performance enhancement induced by $2 \mathrm{D}$ flakes increases with an increase in the hole mobilities of 2D flakes, which is consistent with our experimental results presented in
Fig. 1f. Notably, all conventional HTMs, including SpiroOMeTAD, PTAA, poly(3,4-ethylene dioxythiophene):poly (styrene sulfonate) (PEDOT:PSS), poly(3-hexylthiophene) (P3HT), $\mathrm{NiO}_{x}$ and $\mathrm{CuSCN}$, which are normally used in PSCs, have hole mobilities of less than $0.01 \mathrm{~cm}^{2} \mathrm{~V}^{-1} \mathrm{~s}^{-1}$ (Table S9). In this case, the hole currents from GBs cannot be efficiently conducted out by the HTMs; thus, the advantage of GBs that can form vertical hole channels in PSCs cannot be observed.

\section{Mixed perovskite solar cells modified with 2D flakes}

We then tested the effect of 2D flakes in $\left(\mathrm{CsPbI}_{3}\right)_{0.05}\left(\mathrm{FAPbI}_{3}\right)_{0.95}\left(\mathrm{MAPbBr}_{3}\right)_{0.05}$ mixed PSCs with high efficiency ${ }^{58}$. The mixed PSCs have the normal device structure shown in Fig. 1a. Similarly, different 2D materials dispersed in IPA were spin coated on the perovskite surfaces. Notably, most of the 2D flakes are located on the GBs of perovskite films (Fig. S19a, b). The 2D material interlayer can also form a cascade band structure with the $\left(\mathrm{CsPb}_{3}\right)_{0.05}\left(\mathrm{FAPbI}_{3}\right)_{0.95}\left(\mathrm{MAPbBr}_{3}\right)_{0.05}$ mixed perovskite and spiro-OMeTAD films (Fig. S3h), which will be favourable for charge separation and transport. Similar to the $\mathrm{MAPbI}_{3}$ devices, we find that 2 coats of $2 \mathrm{D}$ flakes on the mixed perovskite leads to the best effect (see Fig. S20).

As shown in Fig. 6a, the pristine mixed PSCs have the best PCE of $20.25 \%, V_{\mathrm{oc}}$ of $1.09 \mathrm{~V}, J_{\mathrm{sc}}$ of $24.56 \mathrm{~mA} \mathrm{~cm}^{-2}$ and $\mathrm{FF}$ of $75.6 \%$, while the BP-modified champion device 

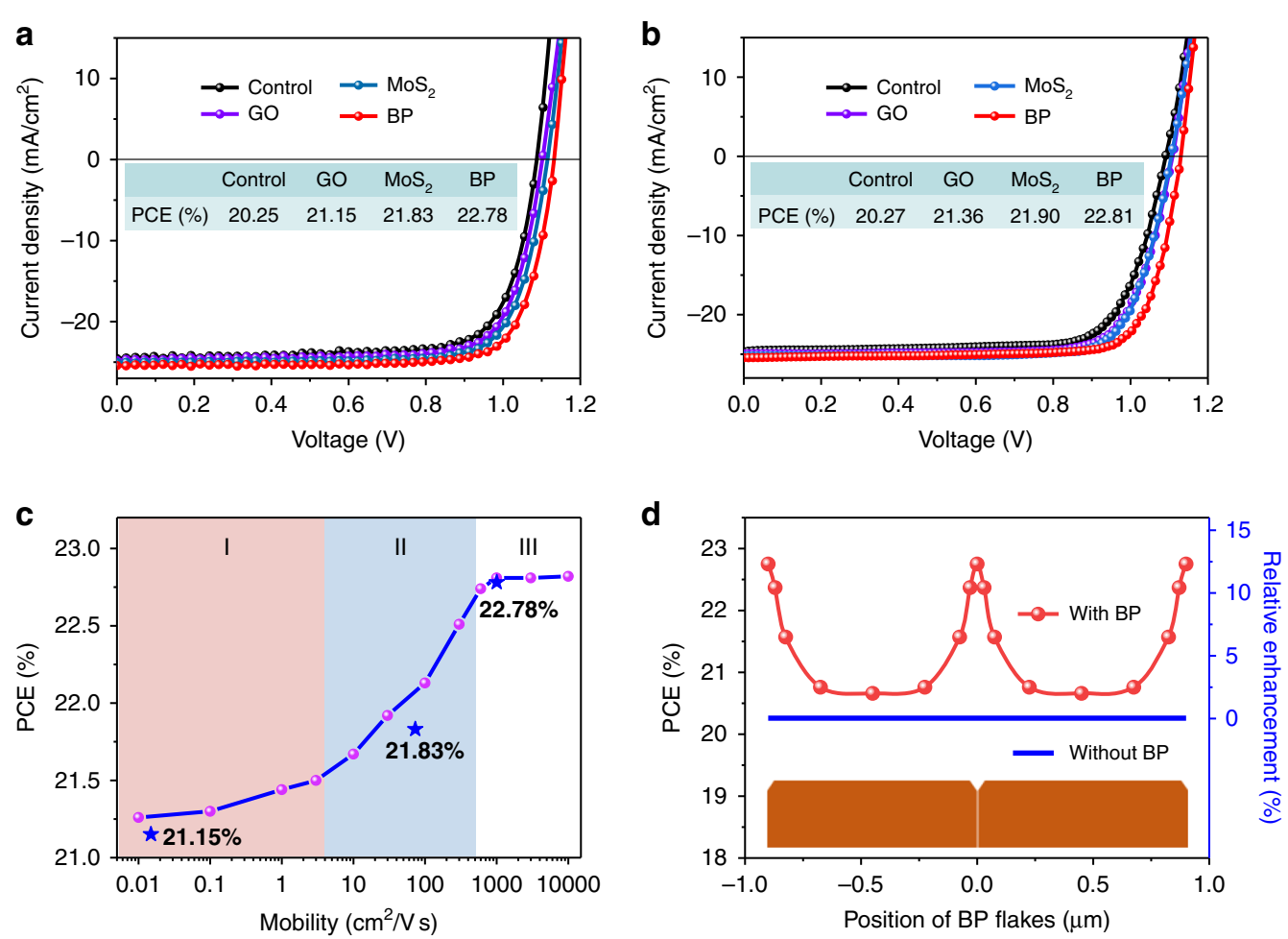

Fig. 6 Photovoltaic performances of the mixed PSCs. $\mathbf{a}, \mathbf{b}$ Experimental (a) and simulated (b) J-V curves of the mixed PSCs modified with different 2D flakes on perovskite GBs. c Simulated device efficiencies of the mixed PSCs with varying mobilities of 2D flakes. The three regions (I, II and III) correspond to the carrier mobilities of the three different 2D flakes (GO, MoS 2 and BP), and the star symbols represent the experimental efficiencies of the PSCs modified with the three different 2D flakes in 6a. $\mathbf{d}$ Simulated device PCEs and efficiency enhancement for BP flakes located at different positions on the perovskite grains. The length of a single BP flake is $\sim 42 \mathrm{~nm}$ in all simulations

exhibits a high PCE of $22.78 \%, V_{\mathrm{oc}}$ of $1.13 \mathrm{~V}, J_{\mathrm{sc}}$ of $25.42 \mathrm{~mA} \mathrm{~cm}^{-2}$ and $\mathrm{FF}$ of $79.3 \%$, with negligible $J-V$ hysteresis $\left(\triangle \mathrm{PCE}=\mathrm{PCE}_{\text {reverse }}-\mathrm{PCE}_{\text {forward }}=0.05 \%\right.$, see Fig. S19c). A stabilized efficiency of $22.5 \%$ is achieved when a bias voltage of $0.96 \mathrm{~V}$ is applied to the champion device (Fig. S19d). Similar to the PSCs based on $\mathrm{MAPbI}_{3}$, the stability of the mixed PSCs was also improved by BP flakes (Fig. S21). For the light stability test, the BPmodified device can maintain $~ 91 \%$ of its initial PCE after light soaking tests for over $400 \mathrm{~h}$, while the control device can only retain $\sim 65 \%$ of its original efficiency (Fig. S21c). It is reasonable to find that the performance enhancements induced by $\mathrm{MoS}_{2}$ and GO flakes are also obvious, and the photovoltaic parameters are shown in Table S10.

As shown in Fig. 6b, mixed PSCs with different 2D flakes are simulated with Silvaco, and the photovoltaic parameters enhanced by the 2D flakes fit very well with the experimental results. Considering that the carrier mobility of a 2D flake varies with its layer number and size, we assumed that the carrier mobilities of 2D flakes changed from 0.01 to $10^{4} \mathrm{~cm}^{2} \mathrm{~V}^{-1} \mathrm{~s}^{-1}$ in the simulation and obtained the relationship between the device efficiency and hole mobility (Fig. S22a and Table S11). As shown in Fig. 6c, regions I, II and III correspond to the carrier mobilities of the three different 2D flakes, namely, $\mathrm{GO}, \mathrm{MoS}_{2}$ and BP, respectively. The device efficiency increases with an increasing carrier mobility, while the enhancement is saturated when the mobility is over $10^{3} \mathrm{~cm}^{2} \mathrm{~V}^{-1} \mathrm{~s}^{-1}$. Therefore, BP should be the optimum 2D material for the conduction of carriers from the GBs in PSCs.

Notably, the performance enhancement induced by BP flakes cannot be simply attributed to the cascade band structure of the perovskite/BP/spiro-OMeTAD interface because the coverage of $2 \mathrm{D}$ flakes on the surface of a perovskite film is less than $10 \%$. To better understand the underlying mechanism, we further simulated the effect of the location of BP flakes on the device performance. Schematic diagrams of the device structures (without BP and with BP flakes at different positions) and corresponding hole current density distribution are shown in Fig. S23. High hole current densities can be found at positions near BP flakes in the simulated mixed PSCs due to enhanced hole conduction at the perovskite/BP/spiroOMeTAD interface. Compared with control devices (without BP), the highest relative PCE enhancement $(\sim 12.5 \%)$ can be obtained when BP flakes are located exactly on GBs (Fig. 6d, Fig. S22b and Table S12), which is 
consistent with our experimental results shown above. In contrast, only an $\sim 2 \%$ relative PCE enhancement can be achieved when the BP flakes are located at the centre of grain surfaces. Therefore, the synergistic effect of BP flakes on GBs in a PSC plays an essential role in performance enhancement.

\section{Discussion}

We developed a convenient and cost-effective approach to modify the GBs of perovskite films by using high-mobility 2D flakes. Although the coverage of 2D flakes on the perovskite films was only several percent, most of the flakes were located on the perovskite GBs. Due to the high carrier mobilities of $2 \mathrm{D}$ materials, especially $\mathrm{BP}$, the hole transfer from GBs was dramatically enhanced in PSCs, resulting in substantial improvements in the efficiency and stability of the devices. Our results also indicated that the GBs in PSCs were not detrimental to the device performance if the accumulated holes in the GBs could be efficiently conducted out. Under certain conditions, GBs could even be favourable for the photovoltaic performance of PSCs due to the built-in electric fields around them, which could facilitate photocarrier separation and transfer in devices. Therefore, the perovskite GBs were electrically benign, which was consistent with previously reported theoretical calculations ${ }^{59-61}$. Notably, we observed the synergistic effect of 2D flakes on GBs in PSCs for the first time. Both the carrier mobility and the location of the $2 \mathrm{D}$ flakes on the perovskite surface were essential to the performance enhancement. This work provides a guideline for modifying perovskite layers with novel high-mobility 2D materials to improve the photovoltaic performance and stability of PSCs.

\section{Materials and methods Materials}

Methylammonium iodide (MAI), formamidinium iodide (FAI), methylammonium bromide $(\mathrm{MABr})$ and $\mathrm{TiO}_{2}$ paste (30 NR-D) were ordered from Greatcell Solar, Ltd, Australia. $\mathrm{PbI}_{2}$ (99.999\%) and dimethylformamide (DMF) were purchased from Alfa Aesar, UK. CsI (99.999\%), dimethylsulfoxide (DMSO), chlorobenzene, titanium isopropoxide, 4-tert-butylpyridine (tBP) and bis(trifluoromethylsulfonyl) imide lithium salt (Li-TFSI) were purchased from Sigma-Aldrich, Ltd, USA. Methylammonium chloride (MACl) and $\mathrm{PbBr}_{2}$ (99.98\%) were purchased from Xi'an Polymer Light Technology Corp, China. Spiro-OMeTAD was purchased from Taiwan Lumtec, Ltd, China.

\section{Preparation of 2D materials}

Commercialized bulk BP crystals were used for the preparation of BP thin flakes. Ten milligrams of the BP crystals were first ground with a mortar in a glovebox, dispersed in $5 \mathrm{ml}$ anhydrous IPA, and then ultrasonicated for $\sim 20 \mathrm{~h}$ with an ultrasonic disrupter (BioSafer 650-92, output power $\sim 400 \mathrm{~W}$ ) at low temperature (maintained by an ice-water bath). The solution was then centrifuged at $3000 \mathrm{rpm}$ for $40 \mathrm{~min}$, and the upper dispersion was collected and re-centrifuged at $6000 \mathrm{rpm}$ for $40 \mathrm{~min}$. The BP thin flakes in the upper dispersion solution were carefully collected for tests and solar cell applications. $\mathrm{MoS}_{2}, \mathrm{GO}$ flakes and BP-QDs were prepared using a similar procedure. ${ }^{51-53}$ The concentration of $2 \mathrm{D}$ flakes was approximately $0.1 \mathrm{mg} / \mathrm{ml}$ (measured by filtration and weighing).

\section{Device fabrication}

PSCs with a normal structure in this work have a configuration of glass/FTO/ $\mathrm{TiO}_{2} /$ perovskite/spiro-OMe$\mathrm{TAD} / \mathrm{Au}$. The FTO substrates $(14 \Omega /$ sq were cleaned with soap water, distilled water, acetone and IPA and treated with $\mathrm{O}_{2}$ plasma for $5 \mathrm{~min}$. A thin $\mathrm{c}-\mathrm{TiO}_{2}$ film $(\sim 30 \mathrm{~nm})$ was then deposited on the FTO glass by spin coating at $4000 \mathrm{rpm}$ from a solution of $0.15 \mathrm{M}$ titanium isopropoxide in ethanol (with the addition of $1.5 \mathrm{mM} \mathrm{HCl}$ from $37 \mathrm{wt} \%$ hydrochloric acid) and was sintered at $500^{\circ} \mathrm{C}$ for $30 \mathrm{~min}$ in an oven. After cooling to room temperature, a $\mathrm{mp}-\mathrm{TiO}_{2}$ layer $(\sim 150 \mathrm{~nm})$ was spin coated on $\mathrm{cp}-\mathrm{TiO}_{2}$ at $4000 \mathrm{rpm}$ by using $\mathrm{TiO}_{2}$ paste (Dyesol 30 NR-D, 30-nm nanoparticles) diluted in tert-butanol (weight ratio 1:7) and sintered again at $500{ }^{\circ} \mathrm{C}$ for $30 \mathrm{~min}$. After cooling down again, the substrates were immersed in a $40 \mathrm{mM}$ clear aqueous solution of $\mathrm{TiCl}_{4}$ for $30 \mathrm{~min}$ at $70^{\circ} \mathrm{C}$, washed with distilled water and IPA, blow-dried with air flow and sintered again at $500^{\circ} \mathrm{C}$ for $30 \mathrm{~min}$. Upon cooling to $150{ }^{\circ} \mathrm{C}$, the substrates were immediately transferred into a glovebox for perovskite film deposition. The perovskite precursor was prepared by dissolving $0.5763 \mathrm{~g}$ of $\mathrm{PbI}_{2}$ and $0.1986 \mathrm{~g}$ of MAI in $1 \mathrm{ml}$ of mixed solvents of anhydrous DMF and DMSO (volume ratio, 8:1). After filtering, $60 \mu \mathrm{l}$ of a $\mathrm{CsPbI}_{3}$ solution $(1 \mathrm{M}$, dissolved in DMF and DMSO, 4:1) was added to the solution. The Csdoped $\mathrm{MAPbI}_{3}$ precursor was spin coated on top of the $\mathrm{TiO}_{2}$ substrates at $4000 \mathrm{rpm}$ for $30 \mathrm{~s}$ in a glovebox. After $10 \mathrm{~s}$ of the spin-coating process, approximately $150 \mu \mathrm{l}$ of chlorobenzene was poured onto the spinning substrate. The substrates were then annealed on a hotplate at $65^{\circ} \mathrm{C}$ for $2 \mathrm{~min}$ followed by $100^{\circ} \mathrm{C}$ for $60 \mathrm{~min}$ in a glovebox. In the experiments of the grain-size effect, the annealing time at $100^{\circ} \mathrm{C}$ was tuned from $10 \mathrm{~min}$ to $120 \mathrm{~min}$. To prepare $\left(\mathrm{CsPbI}_{3}\right)_{0.05}\left(\mathrm{FAPbI}_{3}\right)_{0.95}\left(\mathrm{MAPbBr}_{3}\right)_{0.05}$ mixed perovskite films, $1.013 \mathrm{~g}$ of $\mathrm{FAPbI}_{3}$ yellow powder ${ }^{62}$, $41 \mathrm{mg}$ of $\mathrm{MAPbBr}_{3}$ single crystals ${ }^{63}$ and $38 \mathrm{mg}$ of $\mathrm{MACl}$ were dissolved in mixed solvents of $0.89 \mathrm{ml}$ of DMF and $0.11 \mathrm{ml}$ of DMSO. After filtering, $80 \mu \mathrm{l}$ of a $\mathrm{CsPbI}_{3}$ solution (1 M, dissolved in DMF and DMSO, 4:1) was added to obtain the final mixed perovskite solution. The mixed solution was then spin coated on the $\mathrm{mp}-\mathrm{TiO}_{2}$ films at $1000 \mathrm{rpm}$ for $5 \mathrm{~s}$ and $5000 \mathrm{rpm}$ for $25 \mathrm{~s}$. After $20 \mathrm{~s}$ of the spin-coating process, $0.5 \mathrm{ml}$ ethyl ether was poured onto 
the spinning substrates. The perovskite precursor films were then annealed at $150{ }^{\circ} \mathrm{C}$ for $10 \mathrm{~min}$. For the BPmodified devices, the freshly prepared BP dispersion was spin coated on perovskite films at $4000 \mathrm{rpm}$, followed by annealing at $70^{\circ} \mathrm{C}$ for $5 \mathrm{~min}$. This process may be repeated several times to coat BP flakes with different amounts. After the BP flake modification, a spiroOMeTAD (75 $\mathrm{mg} \mathrm{ml}^{-1}$ in chlorobenzene) solution was spin coated on top at $4000 \mathrm{rpm}$. The spiro-OMeTAD solution $(1 \mathrm{ml})$ was doped with $28.5 \mu \mathrm{l}$ of tBP and $17.5 \mu \mathrm{l}$ of Li-TFSI $\left(520 \mathrm{mg} \mathrm{ml}^{-1}\right.$, dissolved in acetonitrile). Finally, a gold electrode $(\sim 100 \mathrm{~nm})$ was deposited on top through a shadow mask by thermal evaporation. The active area (the overlapping area between the FTO and Au contacts) of an individual solar cell was $8 \mathrm{~mm}^{2}$. Each substrate was then encapsulated with epoxy and glass covers. The fabrication process of PSCs with other 2D materials was similar to that of the BP-modified devices. $\mathrm{MoS}_{2}, \mathrm{GO}$ or BP-QDs dispersions in IPA were spin coated on perovskite films before the deposition of spiroOMeTAD and $\mathrm{Au}$ electrodes. In addition, hole-only devices (ITO/PTAA/MAPbI $3 / \mathrm{Au}$; ITO/PTAA/MAPbI ${ }_{3} /$ $\mathrm{BP} / \mathrm{Au})$ and electron-only devices $\left(\mathrm{ITO} / \mathrm{SnO}_{2} / \mathrm{MAPbI}_{3} /\right.$ $\mathrm{PCBM} / \mathrm{Ag}$; ITO/SnO $/ \mathrm{SAPbI}_{3} / \mathrm{BP} / \mathrm{PCBM} / \mathrm{Ag}$ ) were prepared and tested for space charge limited current (SCLC) measurements.

\section{Material and film characterizations}

AFM measurements were carried out with a Bruker Nanoscope 8 system (tapping mode). TEM observations were carried out by using a JEOL JSM $2100 \mathrm{~F}$ scanning transmission electron microscope operating at $200 \mathrm{kV}$. SEM images were obtained by using a field-emission scanning electron microscope (Hitachi S-4300). FTIR measurements were performed using a Bruker Fourier transform infrared spectroscopy system. UPS measurements were carried out with a VG ESCALAB 220i-XL ultrahigh vacuum surface analysis system equipped with a He-discharge lamp providing He-I photons at $21.22 \mathrm{eV}$. The base vacuum of the system was $\sim 10^{-10}$ Torr, and a $-5.0 \mathrm{~V}$ bias was applied during the measurements. The PL measurements were carried out by using an Edinburgh FLSP920 fluorescence spectrophotometer. A 636-nm laser was used as an excitation light source. The c-AFM measurements were performed by using an MFP-3D series instrument (Asylum Research) with light illumination from the side of the perovskite film. The used AFM tips were coated with $\mathrm{Cr} / \mathrm{Pt}$, and no bias voltage was applied during the test.

\section{Device characterization}

The $J-V$ curves of the PSCs were measured in ambient air with a Keithley 2400 source meter under light illumination of $100 \mathrm{~mW} \mathrm{~cm}^{-2}$ (Newport 91160 solar simulator, $300 \mathrm{~W}$, equipped with an AM $1.5 \mathrm{G}$ filter). The light source was calibrated frequently during the $J-V$ tests by using a standard silicon reference cell (also calibrated) to ensure accurate light intensity. The $J-V$ characteristics were determined by applying an external voltage while measuring the current response. For a standard sweeping cycle, the external applied bias changed from 1.2 to $0 \mathrm{~V}$ (reverse scan) and then returned to $1.2 \mathrm{~V}$ (forward scan) with a voltage scan rate of $30 \mathrm{mV} \mathrm{s}^{-1}$. No preconditioning (such as forward bias or light soaking) was applied before the measurements. EQE spectra were measured with a standard EQE test system from Newport, consisting of a Xenon lamp (Oriel 66902, $300 \mathrm{~W}$ ), a monochromator (Newport 66902), a silicon detector (Oriel 76175_71580) and a dual-channel power meter (Newport 2931_C). EQE measurements were performed in DC mode (300-800 nm) at room temperature. Stable power output characteristics were carried out at the maximum power point of the device under standard light illumination of $100 \mathrm{~mW} \mathrm{~cm}^{-2}$, and the current response was recorded as a function of time by a Keithley 2400 source meter. Impedance measurements of the PSCs were carried out under light illumination of $100 \mathrm{~mW} \mathrm{~cm}^{-2}$ (white light) by using a Zahner Zennium 40630 electrochemical workstation. The oscillating voltage was $50 \mathrm{mV}$, and the applied DC bias voltages varied from 0 to $1.0 \mathrm{~V}$. The frequency of the sinusoidal signal changed from $2 \mathrm{MHz}$ to $1 \mathrm{~Hz}$. For solar cell stability tests, all devices were encapsulated with epoxy/glass.

\section{Device simulation}

Silvaco TCAD 2014 software was used to model and simulate PSCs with a structure of $\mathrm{FTO} / \mathrm{TiO}_{2}(30 \mathrm{~nm}) /$ perovskite $(380 \mathrm{~nm}) /$ spiro-OMeTAD $(120 \mathrm{~nm}) / \mathrm{Au}$ electrode. The device structure and two-dimensional mesh were constructed with the DevEdit module. The output structure file was imported by the ATLAS module followed by setting parameters for user-defined materials and declaring physical models to be calculated. The Luminous module integrated in the ATLAS framework was used to simulate light propagation and absorption. The $J-V$ characteristics of the devices were measured under simulated solar irradiation (AM $1.5 \mathrm{G}, 100 \mathrm{~mW}$ $\mathrm{cm}^{-2}$ ) with light illuminated from the $\mathrm{FTO} / \mathrm{TiO}_{2}$ side. The grain boundaries were simulated by creating a narrow region with a high trap density between two adjacent perovskite grains. We assumed interfacial p-type doping in the GB regions (thickness is $1 \mathrm{~nm}$ ) with a doping level of $10^{15} \mathrm{~cm}^{-3}$ at an energy level of $0.1 \mathrm{eV}$ above the VBM. The thickness of each layer and the dimensions of the grain boundary region are presented in Table S7 and Fig. S23. The recombination of carriers in the body part and at the interfaces was considered by calculating SRH and Auger recombination. Finally, TonyPlot was used to visualize the results written in the $\log$ file output by ATLAS. All diagrams except the $J-V$ curves were 
extracted at a bias voltage of zero. The material parameters used are listed in Table S6 and Table S7.

\section{Acknowledgements}

We acknowledge the funding support from the Research Grants Council of the Hong Kong Special Administrative Region, China (Grant No. PolyU 152087/17E). Z.H. and Y.B.Q. acknowledge the funding support from the Energy Materials and Surface Sciences Unit of the Okinawa Institute of Science and Technology Graduate University, the OIST Proof of Concept (POC) Programme, the OIST R\&D Cluster Research Programme and the JSPS KAKENHI (Grant Number JP18K05266).

\section{Author details}

'Department of Applied Physics, The Hong Kong Polytechnic University, Hung Hom, Kowloon, Hong Kong, China. ${ }^{2}$ College of New Materials and New Energies, Shenzhen Technology University, 518118 Shenzhen, China. ${ }^{3}$ Center of Super-Diamond and Advanced Films (COSDAF), Department of Chemistry, City University of Hong Kong, Hong Kong, China. ${ }^{4}$ Energy Materials and Surface Sciences Unit (EMSSU), Okinawa Institute of Science and Technology Graduate University (OIST), 1919-1 Tancha, Onna-son, Okinawa 904-0495, Japan. ${ }^{5}$ University Research Facility in Materials Characterization and Device Fabrication, The Hong Kong Polytechnic University, Hung Hom, Kowloon, Hong Kong, China

\section{Author contributions}

F.Y. conceived the experiments. P.Y., G.Q.T. and J.P.C. performed the fabrication and characterization of $2 \mathrm{D}$ materials and PSCS. J.P.C. performed the device simulation. N.X.W. assisted with the characterization of device performance. G.Q.T. and C.K.L. tested the hole-only and electron-only devices. D.S., T.W.N. and C.S.L. measured the UPS spectra. Z.H. and Y.B.Q. performed C-AFM measurements of perovskite films. W.L. carried out the TEM experiments. Q.D.T. provided valuable suggestions and discussions. The manuscript was written by P.Y. and F.Y. and discussed and reviewed by all authors. P.Y., G.Q.T. and J.P.C. were equally contributing authors.

\section{Data availability}

The authors declare that the data supporting the findings of this study are available within the paper and its supplementary information files.

\section{Conflict of interest}

The authors declare no competing interests.

Supplementary information The online version contains supplementary material available at https://doi.org/10.1038/s41377-021-00515-8.

Received: 10 October 2020 Revised: 1 March 2021 Accepted: 15 March 2021

Published online: 31 March 2021

\section{References}

1. Kojima, A. et al. Organometal halide perovskites as visible-light sensitizers for photovoltaic cells. J. Am. Chem. Soc. 131, 6050-6051 (2009).

2. National Renewable Energy Laboratory (NREL). Best research-cell efficiency chart. https://www.nrel.gov/pv/cell-efficiency.html. (2021)

3. Miyata, A. et al. Direct measurement of the exciton binding energy and effective masses for charge carriers in organic-inorganic tri-halide perovskites. Nat. Phys. 11, 582-587 (2015).

4. Xing, G. C. et al. Long-range balanced electron- and hole-transport lengths in organic-inorganic $\mathrm{CH}_{3} \mathrm{NH}_{3} \mathrm{Pbl}_{3}$. Science 342, 344-347 (2013).

5. Stranks, S. D. et al. Electron-hole diffusion lengths exceeding 1 micrometer in an organometal trihalide perovskite absorber. Science 342, 341-344 (2013).

6. Shi, D. et al. Low trap-state density and long carrier diffusion in organolead trihalide perovskite single crystals. Science 347, 519-522 (2015).

7. Tai, Q. D. et al. Efficient and stable perovskite solar cells prepared in ambient air irrespective of the humidity. Nat. Commun. 7, 11105 (2016).

8. Jeon, N. J. et al. Compositional engineering of perovskite materials for highperformance solar cells. Nature 517, 476-480 (2015).
9. Yang, W. S. et al. lodide management in formamidinium-lead-halide-based perovskite layers for efficient solar cells. Science 356, 1376-1379 (2017).

10. Said, A. A., Xie, J. \& Zhang, Q. C. Recent progress in organic electron transport materials in inverted perovskite solar cells. Small 15, 1900854 (2019).

11. Gu, P. Y. et al. An azaacene derivative as promising electron-transport layer for inverted perovskite solar cells. Chem. Asian J. 11, 2135-2138 (2016).

12. Seok, S. I., Grätzel, M. \& Park, N. G. Methodologies toward highly efficient perovskite solar cells. Small 14, 1704177 (2018).

13. Zheng, X. P. et al. Defect passivation in hybrid perovskite solar cells using quaternary ammonium halide anions and cations. Nat. Energy 2, 17102 (2017).

14. Shao, Y. C. et al. Origin and elimination of photocurrent hysteresis by fullerene passivation in $\mathrm{CH}_{3} \mathrm{NH}_{3} \mathrm{Pbl}_{3}$ planar heterojunction solar cells. Nat. Commun. $\mathbf{5}$, 5784 (2014)

15. Xu, J. X. et al. Perovskite-fullerene hybrid materials suppress hysteresis in planar diodes. Nat. Commun. 6, 7081 (2015)

16. Bai, Y. et al. Enhancing stability and efficiency of perovskite solar cells with crosslinkable silane-functionalized and doped fullerene. Nat. Commun. 7, 12806 (2016).

17. Son, D. Y. et al. Self-formed grain boundary healing layer for highly efficient $\mathrm{CH}_{3} \mathrm{NH}_{3} \mathrm{Pbl}_{3}$ perovskite solar cells. Nat. Energy 1, 16081 (2016).

18. Liu, Z. K., Lau, S. P. \& Yan, F. Functionalized graphene and other twodimensional materials for photovoltaic devices: device design and processing. Chem. Soc. Rev. 44, 5638-5679 (2015).

19. Tang, G. Q. et al. Solution-phase epitaxial growth of perovskite films on 2D material flakes for high-performance solar cells. Adv. Mater. 31, 1807689 (2019).

20. You, P., Tang, G. Q. \& Yan, F. Two-dimensional materials in perovskite solar cells. Mater. Today Energy 11, 128-158 (2019).

21. Wang, B. et al. The charge carrier dynamics, efficiency and stability of twodimensional material-based perovskite solar cells. Chem. Soc. Rev. 48, 4854-4891 (2019).

22. Agresti, A. et al. Titanium-carbide MXenes for work function and interface engineering in perovskite solar cells. Nat. Mater. 18, 1228-1234 (2019).

23. Zhang, $M$. et al. Synergistic cascade carrier extraction via dual interfacial positioning of ambipolar black phosphorene for high-efficiency perovskite solar cells. Adv. Mater. 32, 2000999 (2020).

24. Wang, Y. et al. Photostability of $\mathrm{MAPb}_{3}$ perovskite solar cells by incorporating black phosphorus. Sol. RRL 3, 1900197 (2019).

25. Chen, W. et al. Black phosphorus quantum dots for hole extraction of typical planar hybrid perovskite solar cells. J. Phys. Chem. Lett. 8, 591-598 (2017).

26. Cao, J. P. et al. Enhanced performance of planar perovskite solar cells induced by van der waals epitaxial growth of mixed perovskite films on $\mathrm{WS}_{2}$ flakes. Adv. Funct. Mater. 30, 2002358 (2020).

27. Yan, F., Migliorato, P. \& Ishihara, R. Simulation of twin boundary effect on characteristics of single grain-silicon thin film transistors. Appl. Phys. Lett. 91, 073509 (2007).

28. Liu, H. et al. Semiconducting black phosphorus: synthesis, transport properties and electronic applications. Chem. Soc. Rev. 44, 2732-2743 (2015).

29. Long, G. et al. Achieving ultrahigh carrier mobility in two-dimensional hole gas of black phosphorus. Nano Lett. 16, 7768-7773 (2016).

30. Li, L. K. et al. Black phosphorus field-effect transistors. Nat. Nanotechnol. 9 372-377 (2014)

31. Hao, C. X. et al. Flexible all-solid-state supercapacitors based on liquidexfoliated black-phosphorus nanoflakes. Adv. Mater. 28, 3194-3201 (2016).

32. Chen, L. et al. Scalable clean exfoliation of high-quality few-layer black phosphorus for a flexible lithium ion battery. Adv. Mater. 28, 510-517 (2016).

33. Youngblood, N. et al. Waveguide-integrated black phosphorus photodetector with high responsivity and low dark current. Nat. Photonics 9, 247-252 (2015).

34. Huang, M. Q. et al. Broadband black-phosphorus photodetectors with high responsivity. Adv. Mater. 28, 3481-3485 (2016).

35. Lin, S. H. et al. Solution-processable ultrathin black phosphorus as an effective electron transport layer in organic photovoltaics. Adv. Funct. Mater. 26, 864-871 (2016)

36. Jeon, N. J. et al. Solvent engineering for high-performance inorganic-organic hybrid perovskite solar cells. Nat. Mater. 13, 897-903 (2014).

37. Bi, E. B. et al. Diffusion engineering of ions and charge carriers for stable efficient perovskite solar cells. Nat. Commun. 8, 15330 (2017).

38. You, P. et al. Efficient semitransparent perovskite solar cells with graphene electrodes. Adv. Mater. 27, 3632-3638 (2015).

39. Lin, P., Yan, F. \& Chan, H. L. W. Improvement of the tunable wettability property of poly(3-alkylthiophene) films. Langmuir 25, 7465-7470 (2009). 
40. Stone, H. A., Aziz, M. J. \& Margetis, D. Grooving of a grain boundary by evaporation-condensation below the roughening transition. J. Appl. Phys. 97, 113535 (2005).

41. Yun, J. S. et al. Benefit of grain boundaries in organic-inorganic halide planar perovskite solar cells. J. Phys. Chem. Lett. 6, 875-880 (2015).

42. Tang, G. Q. et al. Performance enhancement of perovskite solar cells induced by lead acetate as an additive. Sol. RRL 2, 1800066 (2018).

43. Kim, H. S. et al. High efficiency solid-state sensitized solar cell-based on submicrometer rutile $\mathrm{TiO}_{2}$ nanorod and $\mathrm{CH}_{3} \mathrm{NH}_{3} \mathrm{Pbl}_{3}$ perovskite sensitizer. Nano Lett. 13, 2412-2417 (2013).

44. Gonzalez-Pedro, V. et al. General working principles of $\mathrm{CH}_{3} \mathrm{NH}_{3} \mathrm{PbX}_{3}$ Perovskite solar cells. Nano Lett. 14, 888-893 (2014).

45. Liu, J. et al. A dopant-free hole-transporting material for efficient and stable perovskite solar cells. Energy Environ. Sci. 7, 2963-2967 (2014).

46. Wang, Q. et al. Scaling behavior of moisture-induced grain degradation in polycrystalline hybrid perovskite thin films. Energy Environ. Sci. 10, 516-522 (2017).

47. Wang, F. et al. Phenylalkylamine passivation of organolead halide perovskites enabling high-efficiency and air-stable photovoltaic cells. Adv. Mater. 28, 9986-9992 (2016).

48. Ahn, N. et al. Trapped charge-driven degradation of perovskite solar cells. Nat. Commun. 7, 13422 (2016).

49. Han, Q. F. et al. Single crystal formamidinium lead iodide $\left(\mathrm{FAPb}_{3}\right)$ : insight into the structural, optical, and electrical properties. Adv. Mater. 28, 2253-2258 (2016).

50. Sherkar, T. S. et al. Recombination in perovskite solar cells: significance of grain boundaries, interface traps, and defect ions. ACS Energy Lett. 2, 1214-1222 (2017).

51. Nicolosi, V. et al. Liquid exfoliation of layered materials. Science 340, 1226419 (2013).
52. Loh, K. P. et al. Graphene oxide as a chemically tunable platform for optical applications. Nat. Chem. 2, 1015-1024 (2010).

53. Liu, S. H. et al. Black phosphorus quantum dots used for boosting light harvesting in organic photovoltaics. Angew. Chem. Int. Ed. 56, 13717-13721 (2017).

54. Wang, J. L. et al. High mobility $\mathrm{MoS}_{2}$ transistor with low schottky barrier contact by using atomic thick h-BN as a tunneling layer. Adv. Mater. 28, 8302-8308 (2016).

55. Kadantsev, E. S. \& Hawrylak, P. Electronic structure of a single $\mathrm{MoS}_{2}$ monolayer. Solid State Commun. 152, 909-913 (2012).

56. Santos, E. J. G. \& Kaxiras, E. Electrically driven tuning of the dielectric constant in $\mathrm{MoS}_{2}$ layers. Acs Nano 7, 10741-10746 (2013).

57. Chang, H. X. et al. Thin film field-effect phototransistors from bandgap-tunable, solution-processed, few-layer reduced graphene oxide films. Adv. Mater. 22, 4872-4876 (2010)

58. You, P. et al. Ultrafast laser-annealing of perovskite films for efficient perovskite solar cells. Energy Environ. Sci. 13, 1187-1196 (2020).

59. Yin, W. J., Shi, T. T. \& Yan, Y. F. Unusual defect physics in $\mathrm{CH}_{3} \mathrm{NH}_{3} \mathrm{Pbl}_{3}$ perovskite solar cell absorber. Appl. Phys. Lett. 104, 063903 (2014).

60. Guo, Y. G., Wang, Q. \& Saidi, W. A. Structural stabilities and electronic properties of high-angle grain boundaries in perovskite cesium lead halides. J. Phys. Chem. C. 121, 1715-1722 (2017).

61. Walsh, A. et al. Self-regulation mechanism for charged point defects in hybrid halide perovskites. Angew. Chem. Int. Ed. 54, 1791-1794 (2015).

62. Zhang, Y. et al. Achieving reproducible and high-efficiency $(>21 \%)$ perovskite solar cells with a presynthesized FAPbl3 powder. ACS Energy Lett. 5, 360-366 (2020).

63. Saidaminov, M. I. et al. High-quality bulk hybrid perovskite single crystals within minutes by inverse temperature crystallization. Nat. Commun. 6, 7586 (2015). 